\title{
Statistical analysis of numerical preclinical radiobiological data
}

\author{
Joel H. Pitt ${ }^{1}$, and Helene Z. Hill*2 \\ ${ }^{1}$ Renaissance Associates, Princeton, NJ, USA \\ ${ }^{2}$ NJ Medical School, Rutgers University, Newark, NJ 07101-1709, USA \\ *Corresponding author's e-mail address: hill@njms.rutgers.edu
}

Published online: 22 January, 2016 (version 1)

Cite as: Pitt and Hill. ScienceOpen Research 2016 (DOI: 10.14293/S2199-1006.1.SOR-STAT.AFHTWC.v1)

Reviewing status: Please note that this article is under continuous review. For the current reviewing status and the latest referee's comments please click here or scan the QR code at the end of this article.

Primary discipline: Statistics

Secondary discipline: Medicine, Cell biology, Radiology \& Imaging, Life sciences

Keywords Statistical Forensics, Data Fabrication, Tissue Culture, Triplicate Colony Counts, Terminal Digit Analysis, Radiation Biology, Cell Biology

\begin{abstract}
Background: Scientific fraud is an increasingly vexing problem. Many current programs for fraud detection focus on image manipulation, while techniques for detection based on anomalous patterns that may be discoverable in the underlying numerical data get much less attention, even though these techniques are often easy to apply.
\end{abstract}

Methods: We applied statistical techniques in considering and and comparing data sets from 10 researchers in one laboratory and three outside investigators to determine whether anomalous patterns in data from a research teaching specialist (RTS) were likely to have occurred by chance. Rightmost digits of values in RTS data sets were not, as expected, uniform. Equal pairs of terminal digits occurred at higher than expected frequency (>10\%) and an unexpectedly large number of data triples commonly produced in such research included values near their means as an element. We applied standard statistical tests (chi-square goodness of fit, binomial probabilities) to determine the likelihood of the first two anomalous patterns and developed a new statistical model to test the third.

Results: Application of the three tests to various data sets reported by RTS resulted in repeated rejection of the hypotheses (often at $p$-levels well below 0.001) that anomalous patterns in those data may have occurred by chance. Similar application to data sets from other investigators was entirely consistent with chance occurrence.

Conclusions: This analysis emphasizes the importance of access to raw data that form the bases of publications, reports, and grant applications in order to evaluate the correctness of the conclusions and the importance of applying statistical methods to detect anomalous, especially potentially fabricated, numerical results.

\section{INTRODUCTION}

During the past decade, retractions of scientific articles have increased more than 10-fold [1]. At least two-thirds of these retractions are attributable to scientific misconduct: fraud (data fabrication and falsification), suspected fraud, duplicate publication, and plagiarism [2]. Techniques for early identification of fraudulent research are clearly needed. Much current attention has been focused on sophisticated methods for detecting image manipulation [3] and their use is encouraged on the website of the Office of Research Integrity (ORI) of the United States Department of Health and Human Services. But statistical methods which can readily be used to identify potential data fabrication [4-10] are all but ignored by the ORI and the larger world. We believe that routine application of statistical tools to identify potential fabrication could help to avoid the pitfalls of undetected fabricated data just as tools, for example, CrossCheck and TurnItIn, are currently used to detect plagiarism.

The first step in using statistical techniques to identify fabricated data is to look for anomalous patterns of data values in a given data set (or among statistical summaries presented for separate data sets), patterns that are inconsistent with those that might ordinarily appear in genuine empirical data. That such patterns are, indeed, anomalous may potentially be confirmed by using genuine data sets as controls and by using simulations or probabilistic calculations based on appropriate models for the data to show that they would not ordinarily occur in genuine data.

The existence of these anomalous patterns in given suspect data sets may be indicative of serious issues of data integrity including data fabrication [4], but they may also arise as a result of chance. Hence, it is of considerable importance to have statistical methods available to test the hypothesis that a given anomalous pattern in a data set may have occurred as the result of chance.

For example, Mosimann et al. [8] identified instances of fabricated data based on the observation that in experimental data sets containing count data in which the terminal (insignificant) digits are immaterial and inconsequential (hence not under the control of the investigator), it is reasonable to expect and generally the case that these inconsequential digits will 
appear to have been drawn at random from a uniform population. When terminal digits of the count values in a data set of this type do not appear to have been drawn from a uniform population (as may be tested using the chi-square goodnessof-fit test), this may indicate that they have been fabricated.

A test like this is not entirely foolproof. Before applying it, one must ask whether there really is any evidence, beyond mere supposition, that terminal digits of data of the given kind should be random in the sense of uniform. Ideally, one would like to have a probability model for the underlying randomness in the experimental data and use it to show that the distribution of terminal digits of counts values in data sets consistent with that model will be uniform [11]. Alternately, one might be able to run simulations based on an appropriate probability model and demonstrate that the terminal digits of the counts in the simulated data sets do generally appear to have been drawn uniformly. Finally, one could try to validate the assumption that terminal digits of counts in legitimate data sets are uniform, empirically, by testing the uniformity of terminal digits in indisputably legitimate experimental data sets of exactly the same type, constructed using the same protocols, as that of the suspect data.

Simonsohn [10] uncovered fabrications in several psychological research papers based entirely on the summary data available in published reports. He noted that despite the fact that the means of various variables measured in the study varied considerably, their standard deviations were remarkably similar and hypothesized that this would not be the case were the results derived from genuine experimental data. He confirmed his hypothesis with simulation and empirical observation of the distribution of standard deviations in comparable studies.

When we have an appropriate probability model available for the underlying experiment that purportedly produced the suspect data, we can often apply our knowledge of probability theory to determine the probability that an anomalous pattern in question may have occurred by chance in the data set under consideration. Where that probability is less than some reasonable level, we term our tests significant and, in the absence of any alternative explanation, may find any such significant results convincing evidence that the data in question have been fabricated.

\section{THE DATA STUDIED}

Preclinical studies in tissue culture frequently involve clonogenic survival of cells undergoing various treatments [12] and plated in triplicate. We had access to radiobiologic data sets from nine individuals in the same laboratory, which had been accumulated over a span of approximately 10 years. The numerical data generated by one research teaching specialist (RTS) stood apart from the rest when three different statistical tests were applied and compared with the test results of the nine others following the same or similar protocols, as well as when compared with data from three outside laboratories that employed similar techniques. Copies of the laboratory notebooks containing the raw data that we analyzed were in the form of PDF files, which we transferred into Excel spreadsheets. Data are available from the Open Science Framework (https://osf.io) under the search term, "Appendix for Hidden Data" and author names.

We believe that this was a unique situation, as we were able to review and compare essentially all the data from a single laboratory, data produced by a number of independent investigators using the same or similar research techniques, over such a long period of time. In particular, it allowed us to determine whether unusual patterns that we had noted in RTS data sets appeared in the data sets from the other investigators. These patterns in RTS data included (1) a non-uniform distribution of insignificant terminal digits, compared with uniform distributions of such terminal digits in the data of the others; (2) an unusually large frequency of equal terminal digit pairs (i.e., equal rightmost and second rightmost digits) compared with the expected frequency of about $10 \%$ found for the terminal digit pairs in the data of the others; and (3) a surprisingly large number of triplicate colony and cell counts in which a value near the average value of the triple or even that average value itself appeared as one of the constituent counts of the triple, compared with the expected - as determined by a model we developed - such frequencies of average values in the colony and cell counts of others.

We can use the well-known chi-square goodness-of-fit test to determine whether non-uniformity of terminal digits can be considered significant. Additionally, a straightforward test of significance based on the binomial distribution can be used to test the significance of an unusually high frequency of equal terminal digit pairs, but there is no such standard test to determine the significance of unusually large numbers of triplicate counts containing values near their average. Random variation in these triplicate data that are common components of pharmacological, cell biological, and radiobiological experimentation can be analyzed by modeling the triples as sets of three independent, identical Poisson random variables. A major focus of this study is on developing a method to calculate bounds and estimates for the probability that a given set of $n$ such triplicates contains $k$ or more triples which contain their own mean. We use these bounds and estimates in tests of the hypothesis that the observed unusually high incidence of mean containing triples in certain data sets may have occurred by chance.

Our methods should be useful to laboratory investigators in therapeutic, toxicological, cell, and radiation biological studies involving evaluation of clonogenic cell survival after various treatments. Much of our analyses pertain to triple replicates, which are commonly used in cell survival protocols [13-15].

\section{EXPERIMENTAL PROTOCOLS}

The experiments we analyzed followed the same or very similar protocols and employed, with few exceptions, the same Chinese hamster cell line. The cells, harvested from mass culture, were counted, apportioned equally into culture tubes, and incubated overnight with radioisotopes. They were 
washed free of radioactivity and transferred to new tubes for a 3-day incubation at low temperature $\left(10.5^{\circ} \mathrm{C}\right)$ to allow for the given isotope to decay. They were then harvested, triplicate aliquots were suspended for cell counts using a Coulter ZM particle counter, and aliquots were diluted and plated onto tissue culture dishes in triplicate in order that single cells could grow into colonies, which were stained and counted (manually) after about a week.

\section{DATA SETS AND PROBABILITY MODEL}

The primary data sets with which we are concerned are collections of integer Coulter ZM counts (usually in triplicate) and triples of colony counts. The former are copied by hand into a notebook from an LED digital readout of the Coulter ZM counter that counts single cells as they pass randomly through a narrow orifice, the latter are counted by hand. The colony triples are counts of the number of colonies formed by the surviving cells. The counts in each Coulter triple and each colony triple are modeled probabilistically as independent, identical Poisson random variables. The Poisson parameter of these triples will, of course, vary from triple to triple.

Throughout this report, the accumulated data from RTS experiments are independently paralleled to the accumulated data of other investigators including nine members of the laboratory other than RTS who utilized the same Coulter counter and/or counted colonies in the same manner, two professors from out-of-state universities who contributed triplicate data from their Coulter ZM counters, and triplicate colony counts from an additional independent laboratory.

\section{ANALYSIS OF TRIPLICATE DATA}

Many radiobiological experiments result in data sets consisting of triplicate counts where the means of the triples are the key values that are associated with the corresponding treatments in subsequent analyses. This is true, for example, in the generation of survival curves. An investigator wishing to guide the results of such experiments would have to arrange the data

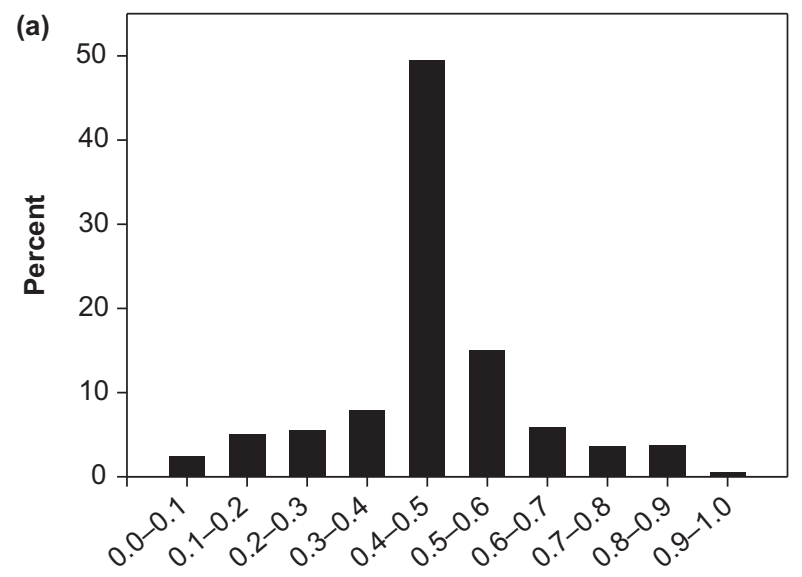

values in each of the triples so that their means are consistent with the desired results. The quickest and easiest way to construct such triples would be to choose the desired mean (or a close approximation) as one of the three count values and then, using two roughly equal constants, calculate the other two values as this initial value plus or minus the selected constants.

Data sets constructed in this manner might then be expected to include either (1) an unusually high concentration of triples whose mid-ratio (the ratio of the difference between the middle value and the smallest value to the difference between the largest value and the smallest value (the gap)) was close to 0.5 or (2) an unusually large number of triples that actually include their own (rounded) mean as one of their values.

\section{Initial mid-ratio review}

Having observed what appeared to us to be an unusual frequency of triples in RTS data containing a value close to their mean, we used $\mathrm{R}$ to calculate the mid-ratios for all of the colony data triples that were available to us. We then constructed histograms of the resulting data sets. The results are shown in Figure 1a and b. The histogram of mid-ratios for RTS colony triples exhibits a distinct predominance of midratios in the range $0.4-0.6$, while the histogram of mid-ratios of the data triples recorded by the nine other members of the laboratory is fairly uniform over the 10 subintervals.

\section{Appearance of the mean in triplicate samples}

We extended our investigation by writing an $\mathrm{R}$ program to identify and count triples that contained their rounded average. Figure 2 is a scan of a page from one of RTS notebooks. Triples that contain their rounded average are highlighted in blue. (In this instance, six of the 10 triples are highlighted.) Of the 1,343 complete colony triples in RTS data, 690 (more than 50\%)

(b)

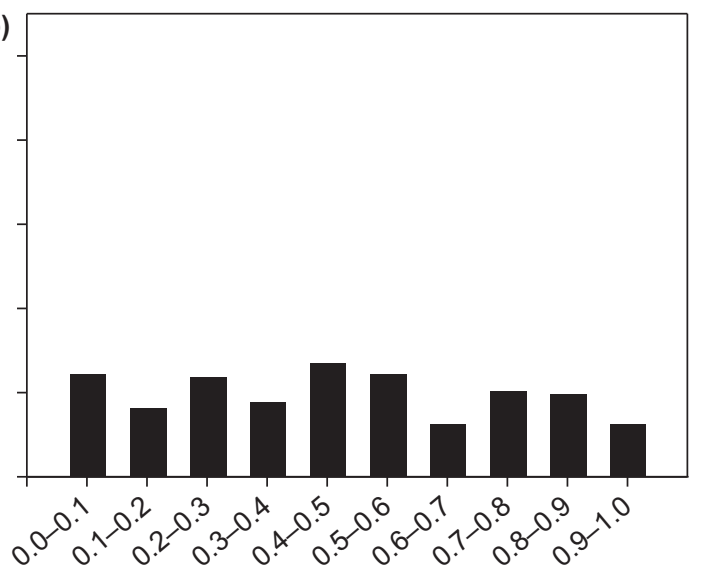

Distribution

Figure 1. Distributions of the mid-ratios (middle-low)/(high-low) for colony triples. (a) RTS, 1343 triples, 128 experiments; (b) other investigators, 572 triples, 59 experiments. 
TABLE-4

Expt \# :
\begin{tabular}{|c|c|c|c|c||c|}
\hline Tube.dilution & Colony 1 & Colony 2 & Colony 3 & Avg Colony & SF \\
\hline 1.2 & 120 & 11 & 99 & 3116.5 & \\
\hline 2.2 & 131 & 121 & 117 & & \\
\hline 3.2 & 104 & 114 & 96 & 104.6 & 0.8984 \\
\hline 42 & 93 & 100 & 86 & 93 & 0.7982 \\
\hline 5.2 & 70 & 78 & 63 & 70.3 & 0.6037 \\
\hline 6.2 & 58 & 68 & 49 & 58.3 & 0.5007 \\
\hline 7.2 & 29 & 22 & 19 & 23.3 & 0.2002 \\
\hline 8.3 & 104 & 115 & 94 & 10.4 & 0.0895 \\
\hline 9.4 & 116 & 126 & 107 & 1.16 & 0.0099 \\
\hline 10.4 & 23 & 29 & 18 & 0.23 & 0.002 \\
\hline
\end{tabular}

Figure 2. PDF image of colony counts from an experiment performed by RTS. The rounded average (highlighted in blue) appears as one of the triplicate counts in six of the 10 samples (ppoibin Prob $=0.00152$ ). For rounding, means with $\geq 5$ after the decimal are rounded up and means with $<5$ after the decimal are rounded down.

contained their rounded average, whereas only 109 (19\%) of the 572 such triples from other investigators did.

Given the marked difference between the percentage of RTS triples that contain their mean and the corresponding percentage of other investigators' triples that do so, and the similar disparity between the histograms of mid-ratios of RTS triples and those of other investigators, it is reasonable to ask whether the apparently excessive numbers of mean/near mean containing triples in RTS data sets might plausibly have occurred by mere chance. In order to answer that question, we used a probability model for such triplicate data to calculate bounds and estimates of the probability that a given set of $n$ such triplicates contains $k$ or more triples. Using these estimates, we are able to test the chance hypothesis.

\section{The model for triplicate data}

The differences between the three actual count values in each colony count triple arise from random differences in the number of cells actually drawn and transferred to the three dishes and the randomness in the numbers of cells that survive the treatment applied to the cells in that triple. As noted above, the random variables that correspond to the number of cells that are originally in each of the three dishes can be modeled probabilistically as the values of three independent, identical Poisson random variables. The common Poisson parameter $\lambda_{0}$ of those three variables will be the (unknown) expected value of those cell counts.
Since the cells in the three dishes have all been exposed to the same level of radiation, the probabilities that a given cell survives to generate a colony should be the same in each of the three dishes. Accordingly, the actual number of survivor colonies in the three dishes will have a binomial distribution with the same $p$ parameter (the common individual cell survival rate) and differing $n$ values corresponding to the numbers of cells on each dish. It is easy to show that these resulting counts have Poisson distributions with parameter $\lambda=\lambda_{0}$ p.

Thus, the three values in each set can be modeled as the values of three independent Poisson random variables sharing a common parameter $\lambda$. The actual value of $\lambda$ varies from triple to triple as it depends both on the specific $\lambda_{0}$ sociated with the initial cell count Poisson distribution and the specific $p$ associated with the treatment that gave rise to the given triple. The likelihood that one of the counts in the triple is equal to or near the triple mean value depends on the value of this parameter.

Given the value of their common Poisson parameter $\lambda$, a relatively straightforward calculation can be used to find the probability that a triple generated by independent, identical Poisson random variables includes its rounded mean (see Appendix). The values of the various $\lambda$ parameters of the Poisson random variables that gave rise to the triples in our data set are, of course, unknown to us, but, in as much as actual colony count values are all less than 400 , we can safely assume that the $\lambda$ parameters of the underlying Poisson random variables are certainly less than 1,000 .

We wrote an $\mathrm{R}$ program to calculate the probability that a triple generated by independent, identical Poisson variables with known parameter $\lambda$ includes its own (rounded) mean value and used it to calculate and create a table (referred to below as the MidProb table) of this probability for all integer values of $\lambda$ from 1 to 2,000 , and as the variation of these probabilities between successive integer values of $\lambda$ greater than 2,000 was negligible, we extended the table by calculating the value of the probability for values of $\lambda$ that were multiples of 100 between 2,100 and 10,000, and multiples of 1,000 between 11,000 and 59,000 (see Table 1 for the first 25 entries). Our calculations showed that as $\lambda$ increased from 1 to 3 , the probability that a randomly generated triple contains its own mean increases from about 0.27 to slightly more than 0.40 and then decreases as $\lambda$ continued to increase. We were thus assured that no matter what the value of $\lambda$ for the Poisson variables that generated a given triple, the probability that the triple would have included its rounded mean as one of its three elements would not exceed 0.42 .

\section{Hypothesis testing I - a nonparametric test}

The observation that the probability that a triple generated by independent, identical Poisson variables with known parameter $\lambda$ includes its own (rounded) mean value never exceeds 0.42 gives us the ability to construct a crude test of the hypothesis that an observed, suspect high number of mean 
Table 1. Partial MidProb table.

\begin{tabular}{llllllllll}
\hline$\Lambda$ & $\boldsymbol{P}$ & $\lambda$ & $\boldsymbol{P}$ & $\lambda$ & $\boldsymbol{P}$ & $\lambda$ & $\boldsymbol{P}$ & $\lambda$ \\
\hline $\mathbf{1}$ & 0.267 & $\mathbf{6}$ & 0.372 & $\mathbf{1 1}$ & 0.317 & $\mathbf{1 6}$ & 0.281 & $\mathbf{2 1}$ & 0.254 \\
$\mathbf{2}$ & 0.387 & $\mathbf{7}$ & 0.359 & $\mathbf{1 2}$ & 0.309 & $\mathbf{1 7}$ & 0.275 & $\mathbf{2 2}$ & 0.250 \\
$\mathbf{3}$ & 0.403 & $\mathbf{8}$ & 0.348 & $\mathbf{1 3}$ & 0.0301 & $\mathbf{1 8}$ & 0.269 & $\mathbf{2 3}$ & 0.246 \\
$\mathbf{4}$ & 0.397 & $\mathbf{9}$ & 0.337 & $\mathbf{1 4}$ & 0.294 & $\mathbf{1 9}$ & 0.264 & $\mathbf{2 4}$ & 0.242 \\
$\mathbf{5}$ & 0.385 & $\mathbf{1 0}$ & 0.327 & $\mathbf{1 5}$ & 0.287 & $\mathbf{2 0}$ & 0.259 & $\mathbf{2 5}$ & 0.238
\end{tabular}

Probability $(P)$ that a triple generated by three independent Poisson random variables with parameter $\lambda$ contains its rounded mean for $\lambda=1-25$. It is clear that $\lambda$ continues to decrease after $\lambda=4$.

containing triples in a given collection of triples may have occurred by chance. Using the number $k$ of triples with gap two or more that contain their means and the number $n$ of triples in the collection, we need to find only the binomial probability $p$ of $k$ or more successes in $n$ independent Bernoulli trials where the probability of success is 0.42 . If the probability $\mathrm{p}$ is less than the chosen $\alpha$ level of the test, we reject the null hypothesis at that significance level. The test is crude in the sense that the calculated $p$ is not the $p$-level of the test, it is simply a (possibly gross) overestimate of the $p$-level. When we apply this test to determine how likely it is that 690 or more of the 1,343 colony triples in RTS data might have contained their rounded average by chance, we find that it less than $2.85 \times 10^{-12}$, an extremely significant result.

Since there are only 109 mean containing triples among the 572 means from other investigators, and 109 is considerably less than the expected number of successes in 572 Bernoulli trials with a success probability of 0.42 , it is immediately clear that the probability of having 109 or more mean containing triples is reasonably large - indeed it is essentially one.

\section{Hypothesis testing II - using $\lambda$ to obtain $p$-values}

It is important to have a more sensitive test, as we can use it to confirm the validity of our model by applying it to what we believe to be legitimate experimental data. To do so, we use a heuristic method to estimate the actual probability that a given collection of $n$ triples includes $k$ mean containing triples. This allows us to provide an actual $p$-value for the one-tailed test we apply for seemingly high numbers of mean containing triples and thereby allows us to determine whether the numbers of mean containing triples in our controls are consistent with our model or whether they are also significantly different from what our model indicates.

We start with the observation that the results of our calculations in the MidProb table show that the probability that a triple of independent, identical Poisson random variables includes its own mean decreases rapidly as $\lambda$ increases. For example, the probability that a triple contains its rounded mean if it is generated by Poisson random variables with $\lambda=20$ is about 0.26 , but with $\lambda=50$ it is less than 0.18 , and with $\lambda=100$ it is less than 0.14 (it even falls to 0.032 when $\lambda=2,000$ ).
We applied a heuristic approach to use our table of calculated values of this probability to estimate (rather than merely bound) the probability that a given collection of $n$ triples that are hypothesized to have been drawn as triples of independent, identical Poisson variables has as many or more than the actual number of mean containing triples than it was observed to contain. We do not know the actual $\lambda$ values of the Poisson random variables that (hypothetically) generated the triples in the data sets, but the mean of any actual triple is a reasonable estimate of the $\lambda$ parameter of the variables that gave rise to it. (The mean is the maximum likelihood estimator in this case.) We can then look up these (rounded) $\lambda$ values in the MidProb table to obtain an estimate of the probability that had the triple been randomly drawn it would contain its own mean.

We are thus able to consider the events that the various individual triples of the collection contain their own means as successes in individual, independent, Bernoulli trials each with a known probability of success. The random variable (statistic) that takes as its value the number of triples in the given data set that contain their own means is the sum of the Bernoulli random variables that indicate success in the various trials. These Bernoulli trials have the known (actually estimated) probabilities of taking the value 1 that we obtain from the MidProb table as described above.

Sums of such independent, not necessarily identically, distributed Bernoulli random variables are said to be Poisson binomial random variables and to have a Poisson binomial distribution. A Poisson binomial random variable that is the sum of $\mathrm{n}$ Bernoulli random variables can potentially take any of the values $0,1, \ldots, n$, and the probability that it takes or is greater than or equal to any of these potential values is completely determined by the probabilities $p_{1}, p_{2}, \ldots, p_{\mathrm{n}}$. that the constituent Bernoulli random variables take the value 1 . Few (if any) standard statistical packages include functions for calculating Poisson binomial distributions. Although there is a straightforward algorithm that can, in principle, be used to calculate probabilities for the distribution function of a Poisson binomial random variable given the success probabilities of the individual Bernoulli variables $p_{1}, p_{2}, \ldots, p_{n}$, issues of numerical stability in these calculations can arise for even moderately large values of $n$, and processing times increase exponentially as $\mathrm{n}$ increases. Nonetheless, we were able to take advantage of an efficient algorithm that has recently been 
developed and implemented as a package for $\mathrm{R}$ [16] to find exact values for the tail $p$-values that we wish to have in testing our null hypothesis.

The function ppoibin in the R package poibin accepts as input two parameters, an integer $j$ and a vector of probabilities $p_{1}, p_{2}, \ldots, p_{\mathrm{n}}$ and returns the probability that the Poisson binomial random variable that corresponds to that vector of probabilities takes a value less than or equal to $j$. To use it to find the probability that there are $k$ or more mean containing triples in a collection of triples generated by groups of three Poisson random variables with common probabilities $p_{1}, p_{2}, \ldots, p_{\mathrm{n}}$ we execute ppoibin with the value $j=k-1$ as the first parameter and the given probabilities as the second and subtract the result from 1 .

We applied this more refined test to RTS collection of 1,343 complete colony triples and found that, given the likely $\lambda$ values that had given rise to the individual triples in the collection, the probability of the observed 690 or more mean containing triples is approximately $6.26 \times 10^{-13}$ (not surprisingly an extremely significant result). Applying the same test to find the probability of finding 109 or more mean containing triples among the 572 complete colony triples that had been recorded by the other investigators in the same laboratory, we found that the probability was 0.47 , and the probability of 109 or fewer such triples is 0.58; results that are entirely consistent with our hypothesis.

\section{Hypothesis testing III - normal estimation of $\boldsymbol{p}$-values}

Given the success probabilities of the individual Bernoulli variables $p_{1}, p_{2}, \ldots, p_{\mathrm{n}}$, the expectation of their Poisson binomial sum is $\mu=\sum_{i=1}^{n} p_{i}$ and its variance $\sigma^{2}=\sum_{i=1}^{n} p_{i}\left(1-p_{i}\right)$. Both are easy to calculate. When the values of the $p_{i}^{\prime} s$ are bounded below, the (Lindeberg-Feller) Central Limit Theorem applies and we can obtain reasonable approximations of the (upper) tail probabilities of a Poisson binomial random variable using normal probabilities.

Where an efficient implementation of an algorithm for calculating exact Poisson binomial probabilities is not available, we can use a normal approximation which with a second-order correction [17] provides a quite precise estimate. Hong [16] reports the results of multiple simulations that indicate that by including the second-order correction the normal approximations to upper tail probabilities will usually - but certainly not always - return probability values marginally higher than the true tail probabilities. The normal distribution we use to approximate a Poisson binomial is the normal with the same mean and standard deviation as the Poisson binomial.

Using the normal approximation has a second advantage, in as much as the $z$-values we calculate in order to look up normal probabilities are informative without recourse to an actual table of normal probabilities. Virtually all students of statistics learn that in normal populations upper tails corresponding to $z$-scores of 2 or more or 3 or more are quite unlikely - with the first having a probability of less than 0.025 and the second having a probability of less than 0.0015 .

To use this approach to approximate the probability of the 690 or more mean containing triples among RTS 1,343 complete triples, we first obtain (to two decimal places) $\mu=220.31$ and $\sigma=13.42$. Using a standard correction for continuity, the $z$-value we use to find the probability of 690 or more mean containing triples is $\frac{689.5-220.31}{13.42}=34.97$ so large that the upper tail probability is effectively indistinguishable from 0 , hence significant at virtually any level.

It is important to keep in mind that the normal distribution probabilities are approximations, not exact values, of the Poisson binomial probabilities. Unfortunately, the normal approximations of upper tail Poisson binomial probabilities are generally less than the true values. In this instance, however, the aforementioned Volkova correction provides the same estimate.

\section{Application to Coulter counts}

While the means of colony triples are the key values of interest to investigators, means of Coulter triples are not as significant. Thus, there is less reason to believe that an investigator wishing to guide results might be inclined to construct Coulter triples that include their own means as one of their values. Nonetheless, we extended our investigation and counted the number of mean containing triples in both RTS Coulter triples and those from other investigators. The results are interesting and illustrate the power and importance of the more sensitive tests we discussed in Sections "Hypothesis testing II - using $\lambda$ to obtain $p$-values" and "Hypothesis testing III - normal estimation of $p$-values" above.

Coulter data from RTS included 1,717 complete triples, 173 of which included their rounded mean, while we had 929 complete Coulter triples from other investigators in the same lab, 36 of which included their rounded means. Application of the crude test described in Section "Hypothesis testing I - a nonparametric test" gives no reason for concern as in both cases the numbers of mean containing triples are consistent with our belief that the probability that any given triple includes its rounded mean will be less than 0.42 .

When, however, we apply the more refined analysis introduced in Sections "Hypothesis testing II - using $\lambda$ to obtain $p$-values" and "Hypothesis testing III - normal estimation of $p$-values", we find reason once again to question RTS data. Coulter count values are in a much higher range than colony count values, and thus, the Poisson random variables that give rise to them have $\lambda$ values in a higher range and probabilities that Coulter triples include their means tend to be lower. Using our table of probabilities, triples of independent Poisson random variables with given $\lambda$ parameters that contain their own mean, we found that were we to randomly generate 1,717 Poisson triples with respective $\lambda$ parameters set equal to the means of RTS actual triples the expected number of mean containing triples would be 97.74 and the standard 
deviation 9.58. Given this (and using the normal approximation to the Poisson binomial), the $7.80 \mathrm{Z}$-score that corresponds to the actual number of 173 mean containing triples in RTS data makes it immediately clear that it is exceedingly unlikely we might have encountered such a large number of mean containing triples by chance. The actual Poisson binomial tail probability is $6.26 \times 10^{-13}$.

When we apply the same analysis to the Coulter triples we obtained from other investigators in the same lab, the results are well within the expected range. According to our calculations the expected number of mean containing triples would be 39.85 and the standard deviation is 6.11 . Hence, the $z$-value corresponding to the actual number of 36 mean containing triples is -0.71 and the actual $p$-value is 0.76 , entirely consistent with our model.

We applied the same analysis to the triplicate Coulter count data sets we had from two investigators in other labs and triplicate colony counts from an investigator in another lab and the results for all of these sets are summarized in Table 2.

\section{Probability model for mid-ratios}

We took a similar approach to evaluating the significance of the occurrence of high percentages of triples having midratios close to 0.5 to that which we used when dealing with triples that contain their mean. In like manner, we wrote an $\mathrm{R}$ function to calculate the probability that the mid-ratio of a triple with a given parameter $\lambda$ falls within the interval $[0.40$, $0.60]$. Using this function, we calculated these probabilities for each of the integer values of $\lambda$ from 1 to 2,000 and stored them in a table. The results of these calculations showed that as $\lambda$ increases from 1 to 10 , the probability that a triple has a mid-ratio in the interval $[0.40,0.60]$ increases from about 0.184 to slightly more than 0.251 and decreases thereafter. Thus, our calculated results tell us that for every value of $\lambda$, the probability that the mid-ratio is in the interval $[0.40,0.60]$ is less than 0.26 . Hence, given a collection of $n$ triples, the probability that $\mathrm{k}$ or more of those triples have mid-ratios in the interval $[0.40,0.60]$ cannot be greater than the probability of $\mathrm{k}$ or more successes in $n$ independent Bernoulli trials in which the probability of success is 0.26 . As was the case when we considered triples which contain their mean, these binomial probabilities can be used to provide a crude but potentially useful test of significance.

We used the same heuristic approach that we had used to develop a more refined significance test for the occurrence of triples that contain their own means to develop a more refined significance test for the incidence of mid-ratios in the $[0.40,0.60]$ interval. This test could be of use in detecting instances in which an investigator wishing to guide the mean values of triplicates employs a reasonably subtle technique.

\section{TERMINAL DIGIT ANALYSIS}

J. E. Mosimann and colleagues $[8,9]$ recommend a technique for identifying aberrant data sets based on the observation that under many ordinary circumstances the least significant (rightmost) digits of genuine experimental count data can be expected to be uniformly distributed and the further observation that when people invent numbers they are generally not uniform.

As per our introductory remarks, it is important to confirm the applicability of this expected uniformity in any context in which we hope to use it. The fact that, in as much as the cells counted in a single batch by the Coulter counter typically number in the several hundreds up to the many thousands, control in selecting the batches of cells to be counted is far from precise enough to extend to the last digit lends some a priori support to the expectation that terminal digits will be uniform. But we also ran simulations generating data sets of triples of independent identical Poisson random variables with comparable means, and the distributions of terminal digits in these sets were consistent with the hypothesis of uniformity. Based on these considerations, we believe it is reasonable to suppose that the Mosiman technique applies to the various Coulter count data sets under consideration. The fact that we are able to apply our tests of uniformity to what we believe to be uncontested experimental data in the course of our test provides further empirical confirmation of the applicability of the Mosiman test.

Table 2. Summary results for analysis of mean containing triples for colony and Coulter count triples from RTS, nine other investigators from the same lab, and investigators in outside labs.

\begin{tabular}{|c|c|c|c|c|c|c|c|c|}
\hline Type & Investigator & No. exps & No. Complete/total & No. mean & No. expected & Sd & $Z$ & $p \geq k$ \\
\hline Colonies & RTS & 128 & $1,343 / 1,361$ & 690 & 220.3 & 13.42 & 34.97 & 0 \\
\hline Colonies & Others & 59 & $572 / 591$ & 109 & 107.8 & 9.23 & 0.08 & 0.466 \\
\hline Colonies & Outside lab 1 & 1 & $49 / 50$ & 3 & 7.9 & 2.58 & -2.11 & 0.991 \\
\hline Coulter & RTS & 174 & $1,716 / 1,717$ & 173 & 97.7 & 9.58 & 7.80 & $6.26 \times 10^{-13}$ \\
\hline Coulter & Others & 103 & $929 / 929$ & 36 & 39.9 & 6.11 & -0.71 & 0.758 \\
\hline Coulter & Outside lab 2 & 11 & $97 / 97$ & 0 & 4.4 & 2.03 & -2.42 & 1.00 \\
\hline Coulter & Outside lab 3 & 17 & $120 / 120$ & 1 & 3.75 & 1.90 & -1.71 & 0.990 \\
\hline
\end{tabular}

Column 3, number of experiments; column 4, number of complete triples with gap $\geq 2$ /total number of triples; column 5 , $\mathrm{k}$, number of mean containing triples; column 6 , number of mean containing triples expected; column 7 , standard deviation of column 6 . 


\section{Application of terminal digit analysis to the data sets}

We counted the number of times each of the digits $0,1, \ldots, 9$ occurred as the rightmost digit of counts copied from the Coulter ZM counter screen and from colony counts. (Note that these analyses do not require that the data be arranged in triplicate sets.) If these least significant digits were indeed uniform - as they should be if the data were truly generated experimentally - then our counts for each of these 10 digits should be roughly the same.

We obtain a more precise measure of the degree to which these distributions diverge from the expected uniform by applying the chi-square test for goodness of fit. We show the actual distribution of terminal digits for the various full data sets we considered in Table 3, along with the computed chisquare statistics and the associated $p$-values. The $p$-values for RTS terminal digit sets result in our rejecting the null hypothesis of uniformity at any reasonable level (and even unreasonable levels) of significance; results for all other investigators' data sets are consistent with our null hypothesis.

\section{EQUAL DIGIT ANALYSIS}

Just as it is reasonable to expect that insignificant terminal digits in experimental data would be approximately uniform, it also seems reasonable to expect that the last two digits of three plus digit experimental data (in which the terminal digits are relatively immaterial) will be equal approximately $10 \%$ of the time. We used $\mathrm{R}$ to count the number of terminal digit pairs in RTS and other investigators' Coulter count data and found that there were 291 (9.9\%) equal pairs of rightmost digit pairs among the 2,942 Coulter count values produced by investigators in the laboratory other than RTS, while there were 636 (12.3\%) such pairs in RTS 5,155 recorded Coulter counts. Assuming that these rightmost pairs were generated uniformly, the probability of 636 or more equal pairs in 5,155 Coulter values is less than $3.3 \times 10^{-8}$, which significantly contraindicates their expected randomness. In contrast, the probability of 291 or more equal pairs among 2,942 Coulter values for the other researchers is 0.587 , which is consistent with our randomness hypothesis.

\section{SUMMARY}

In RTS experiments, the averages of triplicate colony counts appear as one of those counts at improbably high levels based on our model. The rates at which triplicate colony counts reported by other investigators include their averages are consistent with our model.

In RTS experiments, the mid-ratio values of triplicate colony counts fall in the interval $[0.4,0.6]$ at improbably high levels based on our model. The rates at which mid-ratios of triplicate colony counts reported by other investigators fall in that interval are consistent with our model.

Distributions of terminal digits of values in RTS Coulter counts and colonies differ significantly from expected uniformity. This does not hold for the colony and Coulter terminal digits of other workers.

RTS recorded significantly more than the expected one-tenth of the data values for equal terminal digits in Coulter counts. The occurrences and distributions of terminal doubles in the Coulter counts of other workers are close to the expected $10 \%$.

\section{DISCUSSION Limitations}

In most case studies, the number of controls or comparators is either equal to or greater than the number of test values. Since this is a post hoc study, we had no control over the numbers of data we analyzed. To address our concern about smaller comparator sample sizes in one such instance, we randomly selected 314 terminal digits from RTS Coulter results and ran chi-square analyses 100,000 times to test for uniformity. All of the runs would have rejected the null hypothesis for uniformity at the 0.00001 level; one run rejected the hypothesis at the 0.000000001 level. The value of 314 was selected because it is the total number of digits supplied by one of the two outside contributors and was the smallest of the Coulter sample sets with which we worked (cf Table 3).

During the time that RTS was working in the laboratory, few experiments were being performed simultaneously by others, which resulted in some temporal disparity. However, the protocols that we analyzed were followed almost identically

Table 3. Terminal digit analysis of Coulter and colony counts.

\begin{tabular}{|c|c|c|c|c|c|c|c|c|c|c|c|c|c|c|}
\hline \multirow[b]{2}{*}{ Type } & \multirow[b]{2}{*}{ Investigator } & \multicolumn{10}{|c|}{ Digit } & \multirow[b]{2}{*}{ Total } & \multirow[b]{2}{*}{ Chi-square } & \multirow[b]{2}{*}{$P$} \\
\hline & & 0 & 1 & 2 & 3 & 4 & 5 & 6 & 7 & 8 & 9 & & & \\
\hline Coulter & RTS: 174 exps & 472 & 612 & 730 & 416 & 335 & 725 & 362 & 422 & 370 & 711 & 5,155 & 456.4 & 0 \\
\hline Coulter & Others: 103 exps & 261 & 311 & 295 & 259 & 318 & 290 & 298 & 283 & 331 & 296 & 2,942 & 16.0 & 0.067 \\
\hline Coulter & Outside lab-1: 11 exps & 28 & 34 & 29 & 24 & 27 & 36 & 44 & 33 & 26 & 33 & 314 & 9.9 & 0.36 \\
\hline Coulter & Outside lab-2: 17 exps & 34 & 38 & 45 & 35 & 32 & 42 & 31 & 35 & 35 & 33 & 360 & 4.9 & 0.84 \\
\hline Colonies & RTS: 128 exps & 564 & 324 & 463 & 313 & 290 & 478 & 336 & 408 & 383 & 526 & 3,501 & 200.7 & 0 \\
\hline Colonies & Others: 59 exps & 187 & 180 & 193 & 178 & 183 & 173 & 176 & 183 & 183 & 178 & 1,814 & 1.65 & 0.996 \\
\hline Colonies & Outside lab-3: 1 exp & 21 & 9 & 15 & 16 & 19 & 19 & 9 & 19 & 11 & 12 & 150 & 12.1 & 0.21 \\
\hline
\end{tabular}

"Others" refers to other investigators in the laboratory. Outside labs contributed two sets of Coulter data and one set of colony data. Probabilities of 0 were too small to estimate. "Exps" is the number of experiments. 
by all of the members of the laboratory. There is no a priori evidence that the cells, instrumentation, equipment, and consumable supplies used by the other researchers were any different from those utilized by RTS. There is also no evidence that different operators could influence the terminal digits seen on the display of the Coulter counter. All of the investigators used similar techniques to stain and count the colonies.

\section{Power of statistics}

In a recent editorial in Science, Davidian and Louis emphasize the increasing importance of statistics in science and in world affairs as a "route to a data-informed future" [18]. Statistical analysis of numerical data can be used to identify aberrant results [19-21], even in esoteric studies [22,23]. Recently, a rigorous statistical analysis of data that purported to predict the responses to chemotherapeutic agents of human lung, breast, and ovarian cancers demonstrated the erroneous nature of the results [5,24] and led to several retractions [25-28] and a resignation. In this case, patients were potentially directly affected by the use of the wrong drug and/or the withholding of the right drug.

Statistics were used to uncover fraudulent behavior on the part of Japanese anesthesiologist Y. Fujii who is believed to have fabricated data in as many as 168 publications [6]. In like manner, Al-Marzouki et al. [4] used statistics to implicate R.B. Singh for fabricating data in a clinical trial involving dietary habits. Their control, like our comparators, was a similar trial performed using comparable methods by an outside group. Of interest is the fact that Singh was unable to produce his original data for re-examination because it had been, he alleged, consumed by termites. Hudes et al. [7] used statistics to detect unusual clustering of coefficients of variation in a number of articles produced by members from the same biochemistry department in India. The controls for these studies were obtained by searching for similar studies in PubMed. Once data manipulation is suspected, it is up to the statistician to find the proper test(s) to reveal discrepancies to "let the punishment fit the crime," so to speak.

\section{Are RTS data real}

The consistent and highly significant improbability that any of the multiple anomalies observed in RTS data sets are likely to have occurred by chance, and the fact that none of these anomalies appear in any of the many data sets we examined from the nine other investigators in the same laboratory, working under the same conditions with the same equipment or in the comparable data sets we obtained from investigators outside the laboratory, leads us to conclude that RTS data were not obtained in the same manner as the comparator data of others were obtained.

\section{REMEDIES \\ Automated analysis can remove human input into the obtaining of results}

Automatic colony counters are commercially available, and their use in colony survival and other such studies should be encouraged. The counts from particle counters such as the Coulter ZM should be recorded in a form that cannot be altered, such as a printout.

\section{Journals should require the availability and archiving of raw data}

Many now do. This will permit verification, help to avoid unnecessary duplication of experimental results, and facilitate interactions and interchanges among researchers.

\section{Data available}

An excel spreadsheet for performing the proposed analysis is available from Dr Pitt on request, understanding that most researchers performing these types of survival and related experiments are not versed in the use of the statistical program R. Data are available from the Open Science Framework (https://osf.io) under the search term, "Appendix for Hidden Data".

\section{Conclusions}

This analysis emphasizes the importance of access to raw data that form the bases of publications, reports, and grant applications in order to evaluate the correctness of the conclusions and the importance of applying statistical methods to detect anomalous, especially potentially fabricated, numerical results.

\section{REFERENCES}

[1] Van Noorden R. Science publishing: the trouble with retractions. Nature. 2011;478(7367):26-28.

[2] Fang FC, Steen RG, Casadevall A. Misconduct accounts for the majority of retracted scientific publications. Proc Natl Acad Sci USA. 2012;109(42):17028-17033.

[3] Rossner M, Yamada KM. What's in a picture? The temptation of image manipulation. J Cell Biol. 2004; 166(1):11-15.

[4] Al-Marzouki S, Evans S, Marshall T, Roberts I. Are these data real? Statistical methods for the detection of data fabrication in clinical trials. BMJ. 2005;331(7511):267-270.

[5] Baggerly KA, Coombes KR. Deriving chemosensitivity from cell lines: forensic bioinformatics and reproducible research in high-throughput biology. Ann Appl Statist. 2009;3(4): 1309-1334.

[6] Carlisle JB. The analysis of 168 randomised controlled trials to test data integrity. Anaesthesia. 2012;67(5):521-537.

[7] Hudes ML, McCann JC, Ames BN. Unusual clustering of coefficients of variation in published articles from a medical biochemistry department in India. FASEB J. 2009;23(3):689-703.

[8] Mosimann JE, Dahlberg JE, Davidian NM, Krueger JW. Terminal digits and the examination of questioned data. Accountabil Res. 2002;9(2):75-92. 
[9] Mosimann JE, Wiseman DV, Edelman RE. Data fabrication: can people generate random digits? Accountabil Res. 1995;4 (1):31-55.

[10] Simonsohn U. Just post it the lesson from two cases of fabricated data detected by statistics alone. Psychological science (2013): 0956797613480366. Available from http://ssrn.com/abstract= 2114571 or http://dx.doi.org/10.2139/ssrn.2114571

[11] Hill TP, Schürger K. Regularity of digits and significant digits of random variables. Stoch. Proc Appl. 2005;115(10):1723-1743.

[12] Lea DE. Actions of radiation on living cells. London: Cambridge University Press; 1955.

[13] Bonifacino JS. Current protocols in cell biology. New York: John Wiley; 1998. pp. v (loose-leaf).

[14] Katz D, Ito E, Lau KS, Mocanu JD, Bastianutto C, Schimmer AD, Liu FF. Increased efficiency for performing colony formation assays in 96-well plates: novel applications to combination therapies and high-throughput screening. Biotechniques. 2008; 44(2):ix-xiv

[15] Munshi A, Hobbs M, Meyn RE. Clonogenic cell survival assay. In: Blumenthal RD, editor. Miethods in molecular medicine. Totowa NJ: Humana Press; 2005. pp. 21-28.

[16] Hong Y. On computing the distribution function for the sum of independent and non-identical random indicators. Dep. Statit., Virginia Tech, Blacksburg, VA, USA, Tech. Rep. 11-2 (2011).

[17] Volkova AY. A refinement of the central limit theorem for sums of independent random indicators. Theor Probab Appl. 1995;40 (4):791-794.

[18] Davidian M, Louis TA. Why statistics? Science. 2012;336 (6077):12.

[19] Postma E. Comment on "additive genetic breeding values correlate with the load of partially deleterious mutations." Science. 2011;333(6047):1221.

[20] Tomkins JL, Penrose MA, Greeff J, LeBas NR. Additive genetic breeding values correlate with the load of partially deleterious mutations. Science. 2010;328(5980):892-894.

[21] Tomkins JL, Penrose MA, Greeff J, Lebas NR. Retraction. Science. 2011;333(6047):1220.

[22] Brown WM, Cronk L, Grochow K, Jacobson A, Liu CK, Popovic Z, Trivers R. Dance reveals symmetry especially in young men. Nature. 2005;438:1148-1150.

[23] Trivers R, Palestris BG, Zaatari D. The anatomy of a fraud: symmetry and dance. Antioch, CA: TPZ Publishers; 2009.

[24] Baggerly KA, Coombes KR. What information should be required to support clinical "omics" publications? Clin Chem. 2011;57 (5):688-690.

[25] Misconduct in science. An array of errors. The Economist. 2011.

[26] PLoS One prints Potti retraction.... Cancer Lett. 2011;7-8.

[27] Baggerly KA, Coombes KR. Retraction based on data given to Duke last November, but apparently disregarded. Cancer Lett 2010;1:4-6.

[28] Goldberg P. Nevins retracts key paper by Duke group, raising question of harm to patients. Cancer Lett. 2010;36(39):1-4.

\section{COMPETING INTERESTS}

The authors declare no competing interests.

\section{PUBLISHING NOTES}

(C) 2016 Boring et al. This work has been published open access under Creative Commons Attribution License CC BY 4.0, which permits unrestricted use, distribution, and reproduction in any medium, provided the original work is properly cited. Conditions, terms of use and publishing policy can be found at www.scienceopen.com.

Please note that this article may not have been peer reviewed yet and is under continuous post-publication peer review. For the current reviewing status please click here or scan the QR code on the right.

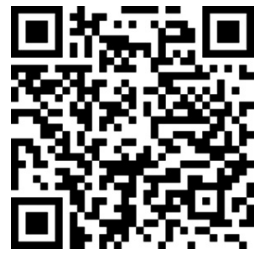

\section{scienceOPEN.com \\ research+publishing network}

\section{APPENDIX \\ Calculating the probability that a Poisson triple contains its rounded mean}

As a preliminary to determining the probability that a triple contains its rounded mean, we first calculated the probability that a triple randomly generated by three independent Poisson random variables with a given $\lambda$ has a gap of two or more and contains its own mean This event is the union of the infinite collection of mutually exclusive events:

$$
\begin{gathered}
A_{j}=\text { the event that the gap is } \mathrm{j} \text { and the triple contains its } \\
\text { own (rounded) mean, for } j=2,3,4,5, \ldots
\end{gathered}
$$

Hence, its probability is the sum of the separate probabilities of the $A_{j}^{\prime} s, \sum_{j=2}^{\infty} P\left(A_{j}\right)$

For each $\mathrm{j}$, the event $A_{j}$ is itself the union of the infinite collection of mutually exclusive events:

$A_{j, k}=$ he event that the largest value in the triple is $\mathrm{k}$ (hence the smallest is $k-j$ ) and the triple includes as one of its elements its own (rounded) mean

where, for any given $\mathrm{j}$, the admissible values of $k$ are $j, j+1$, $j+2, j+3, \ldots$ Hence, $P\left(A_{j, k}\right)=\sum_{k=j}^{\infty} P\left(A_{j k}\right)$

To calculate $P\left(A_{j, k}\right)$, we observe that in order for the event $A_{j, k}$ to toccur, the smallest of the three elements of the triple must be $k-j$, and, of course, the largest must be $k$, but depending on the parity of $j$ there may be one or two different possible values completing the triple. When $j$ is even, the third must be $k-j / 2$ as it is easy to see that this is the only integer value that can complete a triple $\{k-j, n, k\}$ that has mean $\mathrm{n}$. However, when $j$ is odd, there are two distinct integer values that can complete the triple $\{k-j, n, k\}$ so that its rounded mean is $n$; these are $k-[j / 2]$ (where $[x]$ is the greatest integer function, i.e., $[x]=$ greatest integer less than or equal to $x$ ) and $k-[j / 2]-1$.

Since the elements of our triples are assumed to be independently generated Poisson random variables with common parameter $\lambda$, we can obtain formulas in terms of Poisson probabilities for $P\left(A_{j, k}\right)$. We first consider the case $j$ even. Writing $p(n, \lambda)$ for the Poisson probability $\left(e^{-\lambda} \lambda^{n} / n\right)$ of obtaining the value $n$ from a Poisson random variable with parameter $\lambda$, the probability that a triple consists of the values $\{k-j, k-[j / 2], k\}$ in any one of the six different orders in which these numbers can be permuted is $p(k-j, \lambda) p(k-[j / 2], \lambda) p(k, \lambda)$, and hence, the probability of obtaining the triple for $\mathrm{j}$ even is

$$
P\left(A_{j, k}\right)=6 p(k-j, \lambda) p(k-[j / 2], \lambda) p(k, \lambda)
$$


Applying a similar analysis with the two distinct triple types that could result in the event $A_{j k}$ when $j$ is odd, we get for odd $j$

$$
P\left(A_{j, k}\right)=6 p(k-j, \lambda)\left(p(k-[j / 2], \lambda)+p\left(k^{-}[j / 2]-1, \lambda\right)\right) p(k, \lambda)
$$

We combine the preceding observations to obtain a formula for the probability $P(A)$. tt a triplet of numbers chosen independently from the same Poisson distribution contains its (rounded) mean. We get

$$
\begin{gathered}
P(A)=6\left(\sum_{\text {oddj }=3}^{\infty} \sum_{k=j}^{\infty} p(k-j, \lambda)(p(k-[j / 2], \lambda)+p(k-[j / 2]-1, \lambda)) p(k, \lambda)\right. \\
\left.+\sum_{\text {even } j=2 k=j}^{\infty} \sum_{k=j}^{\infty} p(k-j, \lambda) p(k-[j / 2], \lambda) p(k, \lambda)\right)
\end{gathered}
$$

And writing $\operatorname{odd}(x)$ for the function that is 1 when $x$ is odd and 0 when $x$ is even, we can rewrite this as the single double sum:

$$
\begin{aligned}
P(A)= & 6\left(\sum_{j=2}^{\infty} \sum_{k=j}^{\infty} p(k-j, \lambda)(p(k-[j / 2], \lambda)\right. \\
& +\operatorname{odd}(j) p(k-[j / 2]-1, \lambda)) p(k, \lambda))
\end{aligned}
$$

Since we wish to obtain decimal values for these probabilities for various values of $\lambda$, we note that if, for a given $\lambda$ we choose $N$ such that $\sum_{j=N+1}^{\infty} p(j, \lambda)<10^{-9}$. o equivalently, $\sum_{j=0}^{N} p(j, \lambda) \geq 1-10^{-9}$, then we can obtain a value of $P(A)$ accurate to 5 decimal places using the formula:

$$
\begin{aligned}
P(A)= & 6\left(\sum_{j=2}^{N} \sum_{k=j}^{N} p(k-j, \lambda)(p(k-[j / 2], \lambda)\right. \\
& +\operatorname{odd}(j) p(k-[j / 2]-1, \lambda)) p(k, \lambda))
\end{aligned}
$$

Using this formula, we wrote an R program to calculate the probability that a triple of independent Poisson random variables with a common parameter $\lambda$ includes its rounded mean as one of its three elements. We ran this program to create a table of the values of this probability for each of the integer values of $\lambda$ from 1 to 2000 . As a double check on the applicability of our calculation, we performed bootstrap calculations of selected probabilities using $\mathrm{R}$ to perform sets of 200,000 trials. The results were consistent with our calculations. 